Article

\title{
Detection of a Reassortant H9N2 Avian Influenza Virus with Intercontinental Gene Segments in a Resident Australian Chestnut Teal
}

\author{
Tarka Raj Bhatta ${ }^{1,2}{ }^{\oplus}$, Anthony Chamings ${ }^{1,2}$, Jessy Vibin ${ }^{1,2} \oplus$, Marcel Klaassen ${ }^{1,3}(\mathbb{C}$ and \\ Soren Alexandersen $1,2,4, *$ (D) \\ 1 Geelong Centre for Emerging Infectious Diseases, Geelong, Victoria 3220, Australia \\ 2 School of Medicine, Deakin University, Geelong, Victoria 3220, Australia \\ 3 Centre for Integrative Ecology, Deakin University, Victoria 3220, Australia \\ 4 Barwon Health, University Hospital Geelong, Geelong, Victoria 3220, Australia \\ * Correspondence: soren.alexandersen@deakin.edu.au; Tel.: +61-0-342159635
}

Received: 19 December 2019; Accepted: 9 January 2020; Published: 13 January 2020

\begin{abstract}
The present study reports the genetic characterization of a low-pathogenicity H9N2 avian influenza virus, initially from a pool and subsequently from individual faecal samples collected from Chestnut teals (Anas castanea) in southeastern Australia. Phylogenetic analyses of six full gene segments and two partial gene segments obtained from next-generation sequencing showed that this avian influenza virus, A/Chestnut teal/Australia/CT08.18/12952/2018 (H9N2), was a typical, low-pathogenicity, Eurasian aquatic bird lineage H9N2 virus, albeit containing the North American lineage nucleoprotein (NP) gene segment detected previously in Australian wild birds. This is the first report of a H9N2 avian influenza virus in resident wild birds in Australia, and although not in itself a cause of concern, is a clear indication of spillover and likely reassortment of influenza viruses between migratory and resident birds, and an indication that any lineage could potentially be introduced in this way.
\end{abstract}

Keywords: avian influenza virus; low pathogenicity; Chestnut teal; Eurasian lineage; H9N2; phylogenetic analysis; reassortant

\section{Introduction}

Avian influenza virus (AIV) has major direct and indirect impacts on public and veterinary health, as well as wildlife conservation. AIV is a single-stranded, eight-segmented, negative-sense RNA virus in the family Orthomyxoviridae [1]. Each of the gene segments codes for one or more proteins. The virus surface proteins, hemagglutinin (HA) and neuraminidase (NA), are encoded by two separate gene segments. The internal proteins, which include the polymerase basic proteins 1 and 2 (PB1 and $\mathrm{PB} 2$, respectively), polymerase acidic protein (PA), matrix proteins (M1 and M2), nucleoprotein (NP), and nuclear export protein and non-structural protein 1 (NEP-NS1), are encoded by the other six gene segments, respectively [2,3].

Wild ducks and other waterfowl, and to a lesser extent shorebirds and gulls, are considered the main natural reservoir of AIV [4]. AIVs are designated as a low-pathogenicity avian influenza (LPAI) virus or a highly pathogenic avian influenza (HPAI) virus, based on their ability to cause disease in young chickens. This is mediated via a motif of basic amino acids in the HA protein precursor, facilitating proteolytic cleavage by ubiquitous proteases and enabling more generalized tissue infection of HPAI [5-7]. To date, this change from LPAI to HPAI has occurred only in some H5 and H7 subtypes, although in principle it may be possible in other subtypes [8-12]. 
H9N2 AIV is prevalent in poultry and wild birds globally [13]. It has also been reported and isolated from mammalian species like swine [14,15] and humans [16-18], indicating an ability for interspecies transmission. Several studies have shown that some H9N2 viruses have acquired internal genes from highly pathogenic (HP) H5 [19,20] and H7 subtype viruses [21]. These H9N2 viruses may be at an increased risk for becoming a potential pandemic threat [22-24]. While Australia has no land borders, and intercontinental waterfowl migration is highly limited, millions of shorebirds migrate to and from the Australian continent, and may potentially carry with them a variety of AIV subtypes [25-29]. Resident dabbling wild duck species commonly found in wetlands and estuaries in coastal regions of south-eastern Australia, such as Chestnut teals (Anas castanea), share habitats with many migratory shorebirds [30-32], potentially resulting in an exchange of AIVs. [25]. The magnitude of this risk, however, is not known.

Phylogenetically, H9 HA genes are divided into four lineages: h9.1-h9.4. Lineages h9.1 and h9.2 include H9 AIVs isolated in North America. Lineage h9.3 (represented by A/duck/Hokkaido/26/99) contains avian $\mathrm{H} 9$ viruses mainly isolated from Eurasia, but includes some from North America. Lineage h9.4 (G1-like, Y280/G9-like, and Y439-like viruses) solely contains avian H9 strains from Eurasia, and includes viruses that have caused human infection $[14,17,18,33]$. Lineages h9.3 and h9.4 are further divided into sub-lineages [33].

The N2 NA genes have been classified into two lineages, n2.1 and n2.2, which include avian and mammalian influenza viruses, respectively, although viruses within avian sub-lineage n2.1.4 have caused human infections $[16,17,33]$. Lineages n2.1 and n2.2 are further divided into Eurasian and American sub-lineages [33]. The six internal gene segments have also been grouped as Eurasian and American, based on their lineages and sub-lineages [34]. Several studies in Australia have shown the presence of an H9 HA gene segment in combination with an NA gene segment other than N2 in wild birds $[27,28,35]$. Here we describe the first identification and characterization of an H9N2 AIV subtype in a resident wild duck species in southeastern Australia, and conclude that the virus is a typical aquatic bird LPAI virus, with seven gene segments from Eurasian lineage viruses and the NP gene segment from a North American lineage virus.

\section{Materials and Methods}

\subsection{Sample Collection and Ethical Statement}

Fresh faecal samples were collected from five wild-caught Chestnut teals older than one year in age on 13 August 2018 in Wallington, Victoria, Australia. These samples were frozen at $-80{ }^{\circ} \mathrm{C}$ within 1-3 $\mathrm{h}$ of collection. The five faecal samples were initially processed for nucleic acid extraction as a pool, designated as pooled sample CT08.18. AIV was subsequently detected in two out of five cloacal and oropharyngeal swab samples collected from the same five individual birds by a matrix gene PCR performed at the World Health Organization (WHO) Collaborating Centre for Reference and Research on Influenza, Melbourne, Australia. The two corresponding individual faecal samples (samples 11356 and 12952) were later also processed individually, but after another 11 months of storage at $-80^{\circ} \mathrm{C}$. The bird sample collection was approved under Deakin University's Animal Ethics Committee project number B43-2016, as well as Department of Environment, Land, Water and Planning permit number 1008206.

\subsection{Virus Enrichment, Nucleic Acid Extraction, cDNA Synthesis, and Non-Targeted Amplification of Pooled and Individual Samples}

All samples, both pooled and individual, were processed, and virus particles were enriched using a protocol described previously [36,37]. Briefly, the samples were homogenised at $25 \mathrm{~Hz}$ for $2 \mathrm{~min}$, followed by centrifugation at $17,000 \times \mathrm{g}$ for $3 \mathrm{~min}$, and the supernatant was filtered using a $0.8 \mu \mathrm{m}$ polyethersulfone (PES) spin-filter. The samples were then divided into two, one aliquot ultracentrifuged at $178,000 \times g$ for $1 \mathrm{~h}$ and the other not ultracentrifuged. Both of the aliquots were 
then treated with benzonase and micrococcal nuclease for $2 \mathrm{~h}[36,37]$. The ultracentrifuged samples were then treated with $200 \mu \mathrm{M}$ PMAxx for $30 \mathrm{~min}$ in a PMA-Lit LED Photolysis Device, as indicated by the manufacturer (Biotium, Fremont, CA, USA). Finally, nucleic acids were extracted using the QIAamp Viral RNA Mini Kit (Qiagen, Hilden, Germany). The cDNA synthesis and DNA amplification were carried out using the SeqPlex RNA Amplification Kit (Sigma, St. Louis, MO, USA), as per the manufacturer's protocol [36,37].

\section{3. cDNA Synthesis and Targeted Avian Influenza Virus PCR Amplification from Individual Samples}

cDNA was synthesized from the individual chestnut teal samples using $5 \mu \mathrm{L}$ of nucleic acids (obtained after virus enrichment, as described above) as the starting material, using $10 \times$ PathAm FluA RT Enzyme Mix at $45^{\circ} \mathrm{C}$ for $60 \mathrm{~min}$, and then at $95^{\circ} \mathrm{C}$ for $1 \mathrm{~min}$, as described in the manufacturer's protocol (Applied Biosystems, Foster City, CA, USA). Synthesized cDNA was then amplified using $25 \times$ PathAmp FluA PCR Primer Mix and 5 U/ $\mu$ L SuperTaq plus PCR Enzyme (Applied Biosystems) at $95^{\circ} \mathrm{C}$ for $4 \mathrm{~min}$, with 40 cycles of $95^{\circ} \mathrm{C}$ for $15 \mathrm{~s}, 55^{\circ} \mathrm{C}$ for $30 \mathrm{~s}$, and $68^{\circ} \mathrm{C}$ for $2 \mathrm{~min}$, with a final $68^{\circ} \mathrm{C}$ step for 7 min using an Applied Biosystems ProFlex PCR system (Thermofisher Scientific, Waltham, MA, USA). The amplified products were purified using 1.5× Agencourt AMPure XP kit (Beckman Coulter, Brea, CA, USA), and $5 \mu \mathrm{L}$ of the purified products were then fragmented and amplified using the same SeqPlex Kit (Sigma), as described above, following the manufacturer's protocol [36,37].

\subsection{Library Preparation, Next Generation Sequencing (NGS), and Detection of Avian Influenza Virus Sequences}

Library preparation was performed using the Ion Plus Fragment Library Kit and IonXpress Barcode Adapters 1-96 Kit (Thermo Fisher Scientific). The libraries were quantified using the Ion Library TaqMan Quantitation Kit (Thermo Fisher Scientific), and then pooled. Sequencing was performed using Ion 530 chips and an Ion Torrent S5XL System (Thermo Fisher Scientific). Generated sequence reads were compared to a local database of virus sequences downloaded from Genbank (December 2018) using BLASTN $[38,39]$, with an e-value cut-off score of $1 \times 10^{-10}$, and a BLASTX $[38,39]$ query with an e-value cut-off of $1 \times 10^{-10}$. After the initial detection of AIV in the pooled sample, the same libraries were run a second time, using an Ion 540 chip to increase the amount of sequence generated. Both the non-targeted and targeted amplified individual samples were sequenced using Ion 530 chips.

\subsection{Alignment of Avian Influenza Virus Reads and Phylogenetic Analysis}

The sequence data generated from pooled and individual samples were analysed for the presence of AIV sequences. The AIV sequence reads from non-targeted pooled and individual samples were aligned using MEGA 7 software [40], because of very few AIV reads present and partial gene sequences created for each of the eight different AIV genome segments. For the targeted AIV PCR-amplified individual samples, reads were mapped to the nearest reference genomes using the TMAP plugin [41] and a mapping quality score of 80 or higher. Consensus sequences were generated in Integrative Genomics Viewer software (IGV) (Broad Institute, Cambridge, MA, USA) [42]. The final consensus was run as a reference in the TMAP plugin [41] to determine an accurate read depth coverage.

Generated sequences were compared with the online NCBI Genbank database using BLASTN [38,39], and the closest, as well as additional related or representative sequences of significant clades from around the globe, were selected for further analyses. Selected sequences were aligned using Clustal-W [43] and phylogenetic trees, created using the Maximum Likelihood (ML) method with the best fitting model, as determined by MEGA 7 [40]. The robustness of different phylogenetic nodes was assessed using 1000 bootstrap replicates. 


\subsection{Attempt at Virus Isolation}

Virus isolation was attempted on the two influenza PCR-positive individual samples in embryonated chicken eggs at the Australian Animal Health Laboratory (AAHL), Geelong.

\section{Results}

\subsection{Detection of Avian Influenza Virus Sequences by Next-Generation Sequencing, Using the} Non-Targeted Method

The pooled CT08.18 sample generated about 10 million reads in the first NGS run, of which 40 reads (corresponding to $0.0004 \%$ of total reads) mapped to AIV. Rerunning the same NGS libraries using a 540 chip generated another 31 million reads, of which 129 reads $(0.0004 \%$ of total reads) mapped to AIV, resulting in a total of 169 reads and covering partial sequences of all eight gene segments of AIV from the pooled sample CT08.18 (Table S1). These 169 reads came from the sample for which the enrichment included ultracentrifugation, consistent with the fresh, pooled sample containing complete AIV virions.

For the non-targeted individual samples, individual sample 11356 generated around 3.8 million reads, of which only four reads ( $0.0000014 \%$ of total reads) mapped to AIV. Individual sample 12952 generated around 13.7 million reads of which only 21 reads $(0.0000015 \%$ of total reads) mapped to AIV. Interestingly, and in contrast to the pooled sample mentioned above, most of these AIV reads (19 of 25) came from the non-ultracentrifuged samples, indicating that AIV virions may have deteriorated during the prolonged storage. Only reads mapping to the NP gene segment were obtained from the individual sample 11356, whereas a very few reads (five for PB2, one for PB1, one for PA, three for HA, four for NP, five for NA, one for M1-M2, and one for NEP-NS1) were mapped to each of the eight gene segments in the individual sample 12952.

\subsection{Detection of Avian Influenza Virus Sequences by Next-Generation Sequencing Using the Targeted Avian} Influenza Virus PCR Amplification Method

In the two individual samples where the PathAmp Flu A influenza virus amplification was performed, individual sample 11356 generated around 73,000 reads, of which 1500 reads ( $2 \%$ of total reads) mapped to AIV; sample 12952 generated around 81,000 reads, of which more than 28,000 reads ( $\sim 35 \%$ of total reads) mapped to AIV. Out of the eight AIV gene segments, we obtained partial sequences of six gene segments, except for PB1 and PA, in individual sample 11356, while for sample 12952 we obtained the full length sequences of these same six segments. Segments PB1 and PA were not found to be amplified in either of these samples. Similar to what we observed for the individual non-targeted samples, more AIV reads were obtained from the non-ultracentrifuged preparations, and only very few from the ultracentrifuged ones.

\subsection{Phylogenetic Analysis and Comparison of Avian Influenza Virus Gene Segments}

The six full consensus gene segment sequences from AIV targeted sequencing of individual sample 12952, together with concatenated PB1 and PA gene segment sequences from the pooled sample CT08.18, were selected for phylogenetic analysis. Detailed analysis of the obtained sequences and comparison with the reference sequences identified an H9N2 subtype AIV in our pooled and individual samples. The virus from the pooled sample was named as A/Chestnut teal/Australia/CT08.18/2018 (H9N2). Similarly, the virus from individual sample 12952 was named as A/Chestnut teal/Australia/CT08.18/12952/2018 (H9N2). Due to the few partial sequences obtained from sample 11356, results from this sample were not included in any phylogenetic trees. However, obtained partial AIV sequences from the amplified individual sample 11356 were found to be identical with the amplified individual sample 12952. Similarly, the limited partial sequences obtained from individual sample 12952 without influenza virus PCR amplification were found to be $100 \%$ identical to the 12952 influenza virus with PCR amplification. The respective gene segment sequences in the 
pooled sample were found to be almost identical (>99.5-99.9\%) in five of the six segments, while the sequences for the matrix gene segment was found to be $100 \%$ identical.

\subsection{Identification of Avian Influenza Virus Gene Segment Lineages}

The highest nucleotide sequence identities for seven of the eight gene segments on Genbank were found to be Eurasian lineage AIVs from different species of wild birds. The NP gene segment showed 98.12\% similarity to a North American lineage AIV, as shown in Tables 1 and 2.

Table 1. Avian influenza virus (AIV) isolates from GenBank with the highest nucleotide identities to complete gene segments of A/Chestnut teal/Australia/CT08.18/12952/2018 (H9N2).

\begin{tabular}{|c|c|c|c|c|c|}
\hline Gene Segment & $\begin{array}{c}\text { Genbank Virus Sequence with Highest } \\
\text { Nucleotide Identity }\end{array}$ & Accession Number & Sub-Lineages * & Nucleotide Match & Identity $\%$ \\
\hline PB2 & A/duck/Bangladesh/26992/2015 (H7N9) & KY635525 & $\begin{array}{c}\text { S1.2.4 } \\
\text { (Eurasian) }\end{array}$ & $2280 / 2308$ & 98.79 \\
\hline HA & A/Grey Teal/Victoria/GT001/2017 (H9N1) & MK213322 & $\begin{array}{c}\text { h9.3.3 } \\
\text { (Eurasian) }\end{array}$ & $1667 / 1703$ & 97.89 \\
\hline NP & A/Ruddy Turnstone/MW02/Tas/2014 (H10N8) & MH453824 & $\begin{array}{l}\text { S5.1 (North } \\
\text { American) }\end{array}$ & $1517 / 1546$ & 98.12 \\
\hline NA & A/duck/Mongolia/154/2015 (H1N2) & LC121278 & $\begin{array}{l}\mathrm{n} 2.1 .6 \\
\text { (Eurasian) }\end{array}$ & $1441 / 1463$ & 98.49 \\
\hline M1-M2 & A/Grey Teal/Victoria/GT001/2017 (H9N1) & MK213323 & $\begin{array}{c}\text { S7.2.5 } \\
\text { (Eurasian) }\end{array}$ & $969 / 982$ & 98.68 \\
\hline NEP-NS1 & A/Ruddy Turnstone/MW02/Tas/2014 (H10N8) & MH453825 & $\begin{array}{c}\text { S8.2.1 } \\
\text { (Eurasian) }\end{array}$ & $872 / 882$ & 98.87 \\
\hline
\end{tabular}

*: "S" stands for sub-lineage; " $\mathrm{h}$ " and " $\mathrm{n}$ " stands for hemagglutinin and neuraminidase sub-lineages, respectively.

Table 2. AIV isolates from GenBank with the highest nucleotide identities to partial gene segments of A/Chestnut teal/Australia/CT08.18/2018 (H9N2).

\begin{tabular}{|c|c|c|c|c|c|}
\hline Gene Segment & $\begin{array}{c}\text { Genbank Virus Sequence with Highest } \\
\text { Nucleotide Identity }\end{array}$ & Accession Number & Sub-Lineages * & Nucleotide Match & Identity $\%$ \\
\hline PB1 & A/duck/Mongolia/154/2015 (H1N2) & LC121274 & $\begin{array}{c}\text { S2.2.2 } \\
\text { (Eurasian) }\end{array}$ & $1338 / 1381$ & 96.88 \\
\hline PA & A/Grey Teal/Victoria/GT001/2017 (H9N1) & MK213327 & $\begin{array}{l}\text { S3.2.7 } \\
\text { (Eurasian) }\end{array}$ & $1120 / 1130$ & 99.12 \\
\hline
\end{tabular}

*: "S" stands for sub-lineage.

\subsection{Phylogenetic Analysis of the H9 Gene Segment}

A 1718 nt long, full consensus sequence of the H9 HA gene segment (Genbank accession: MN826601) was obtained from the individual sample 12952, with a coverage depth ranging from 3 to 1272 at a mapping quality threshold of 80 or higher. Phylogenetic analysis of this segment was performed together with a total of 44 representative $\mathrm{H} 9$ reference sequences selected from the NCBI GenBank database. A/Chestnut teal/Australia/CT08.18/12952/2018 (H9N2) was most similar in nucleotide sequence (approximately 98\%) to A/Grey Teal/Victoria/GT001/2017(H9N1) from Australia [28] (Genbank accession: MK213322), and belongs to Eurasian sub-lineage h9.3.3 [33,44], as shown in Figure 1. Five other H9 gene segment sequences previously reported from Australian birds from 2007-2012 also belong to sub-lineage h9.3.3, although these were only 91-93\% identical to A/Chestnut teal/Australia/CT08.18/12952/2018 (H9N2). The HA cleavage site of A/Chestnut teal/Australia/CT08.18/12952/2018 (H9N2) was found to posess the amino acid sequence ASDR/GLF, confirming this as a low pathogenicity strain. 


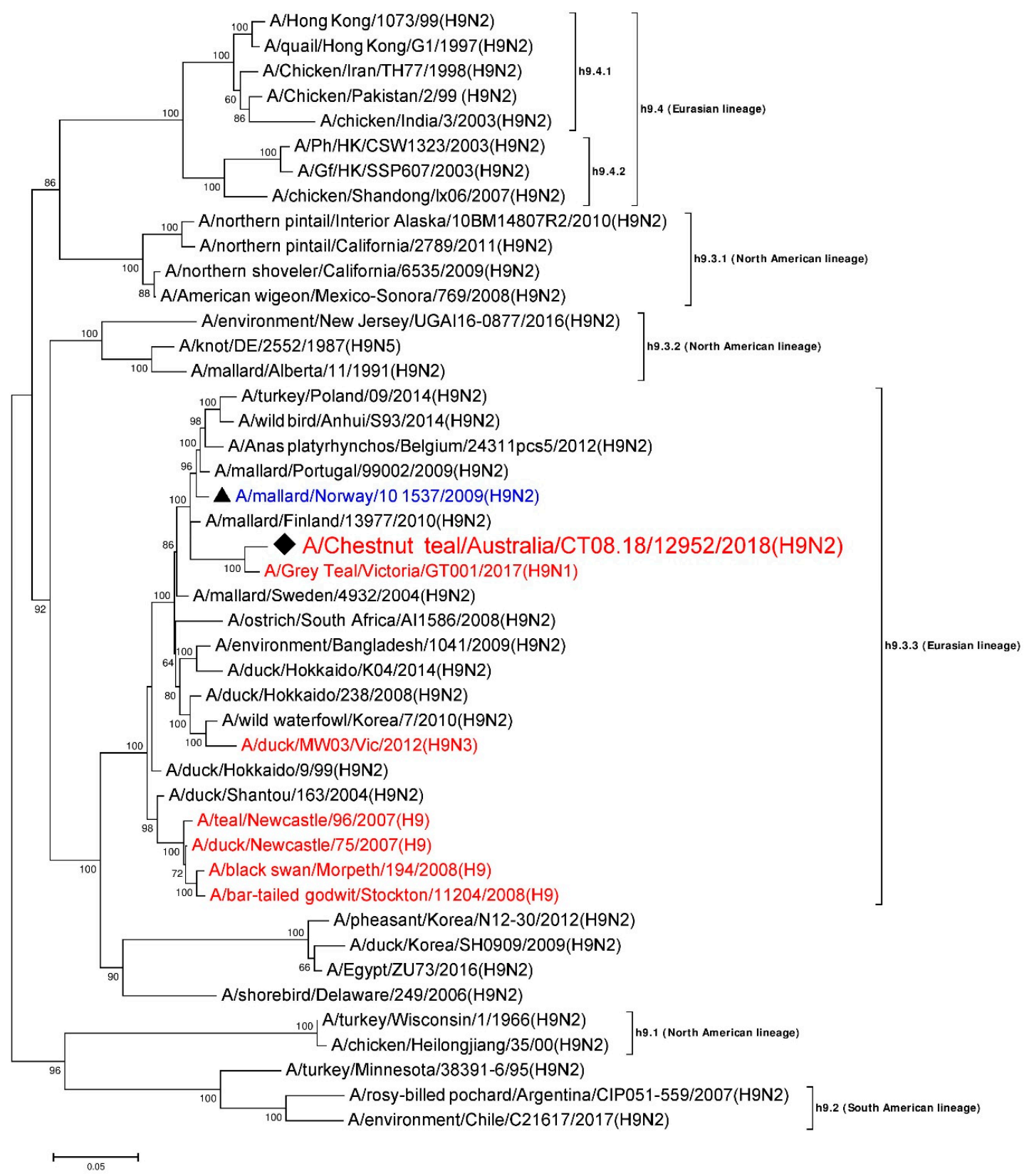

Figure 1. Phylogenetic analysis of the $\mathrm{H} 9$ gene segment. The nucleotide sequences were aligned and analyzed using the maximum likelihood method in MEGA 7.0 [40], using the Tamura-Nei (TN93+G) [45] model with a bootstrapping of 1000 replicates. The analysis involved 45 sequences of the $\mathrm{H} 9$ gene segment of influenza virus, including A/Chestnut teal/Australia/CT08.18/12952/2018 (H9N2) from a resident Chestnut teal. The numbers at the nodes represent bootstrap values, and only bootstrap values at or above $60 \%$ are shown. Branch lengths are scaled according to the number of nucleotide substitutions per site. The sequence from the current study has been labelled with a black rhombus ( $\downarrow)$, while a representative reference sequence from Eurasian lineage has been labelled with a black triangle (ム) with text in blue color, and is shown on all figures for easy reference. Red colored sequences are from AIVs detected in Australia.

\subsection{Phylogenetic Analysis of the N2 Gene}

A 1466 nt long, full consensus sequence of the N2 NA gene segment (Genbank accession: MN826603) was obtained from the individual sample 12952, with a coverage depth ranging from 2 to 2585 and at a mapping quality threshold of 80 or higher. Phylogenetic analysis of this segment was performed, together with a total of $37 \mathrm{~N} 2$ representative reference sequences selected from the 
NCBI GenBank database. A/Chestnut teal/Australia/CT08.18/12952/2018 (H9N2) was most similar in nucleotide sequence (>98\%) to A/duck/Mongolia/154/2015 (H1N2) (Genbank accession: LC121278), and belongs to the n2.1.6 Eurasian lineage [33,44], as shown in Figure 2. The N2 sequences from the American lineages formed separate clusters. To the best of our knowledge, this is the first report of an $\mathrm{N} 2$ gene sequence from an Australian bird. The other reference sequences included in Figure 2 were found to be $\sim 85-98 \%$ identical to A/Chestnut teal/Australia/CT08.18/12952/2018 (H9N2).

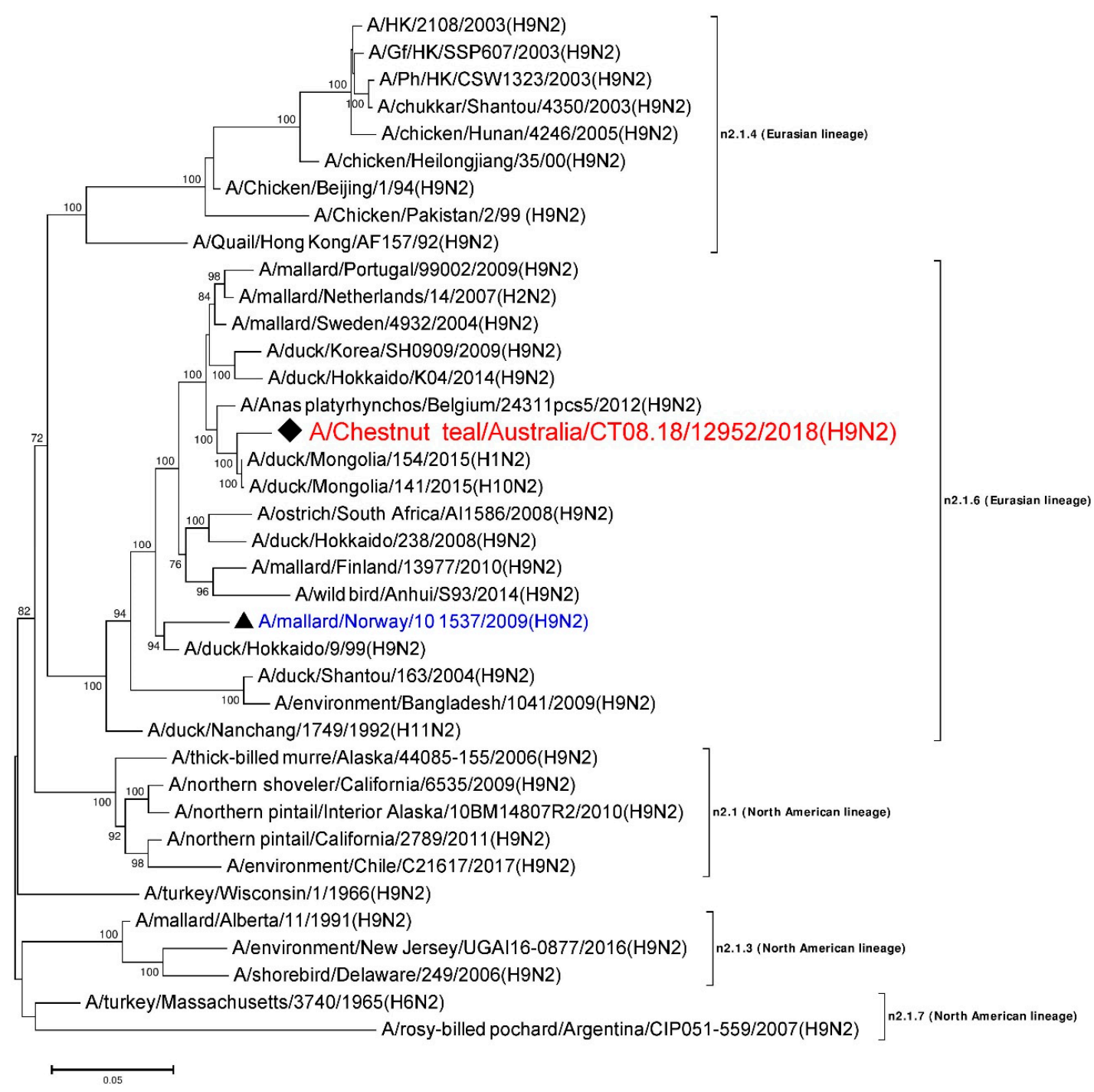

Figure 2. Phylogenetic analysis of the N2 gene segment. The nucleotide sequences were aligned and analyzed using the maximum likelihood method in MEGA 7.0 [40], using the Tamura three-parameter $(\mathrm{T} 92+\mathrm{G})[46]$ model with a bootstrapping of 1000 replicates. The analysis involved 38 sequences of the N2 gene segment of influenza virus, including A/Chestnut teal/Australia/CT08.18/12952/2018 (H9N2), from a resident Chestnut teal. The numbers at the nodes represent bootstrap values, and only bootstrap values at or above $60 \%$ are shown. Branch lengths are scaled according to the number of nucleotide substitutions per site. The sequence from the current study has been labelled with a black rhombus ( $\downarrow)$, while a representative reference sequence from Eurasian lineage has been labelled with a black triangle $(\boldsymbol{\Lambda})$, with text in blue color, and is shown on all figures for easy reference. Red colored sequences are from AIVs detected in Australia.

\subsection{Phylogenetic Analysis of the Nucleoprotein Gene}

A $1550 \mathrm{nt}$ long, full consensus sequence of NP gene segment (Genbank accession: MN826602) was obtained from the individual sample 12952, with a coverage depth ranging from 3 to 703, at a 
mapping quality threshold of 80 or higher. Phylogenetic analysis of this segment was performed, together with a total of $36 \mathrm{NP}$ representative sequences selected from the NCBI GenBank database. A/Chestnut teal/Australia/CT08.18/12952/2018 (H9N2) was most similar in nucleotide sequence (>98\%) to A/RuddyTurnstone/MW02/Tas/2014(H10N8) [28] from Australia (Genbank accession: MH453824). A/RuddyTurnstone/MW02/Tas/2014(H10N8) [28] belonged to sub-lineage S5.1, the avian NP lineage from North America from the 1960s-2000s [34,44,47] (Figure 3). The NP gene segment from other wild birds from Australia from 2012-2017 also fell in the same lineage S5.1, with around 97-98\% nucleotide identity to A/Chestnut teal/Australia/CT08.18/12952/2018 (H9N2). In contrast, the NP gene from Australian wild birds from 1983 made a separate cluster as lineage S5.7, which is separate again from the Eurasian lineage S5.2.1, as shown in Figure 3.

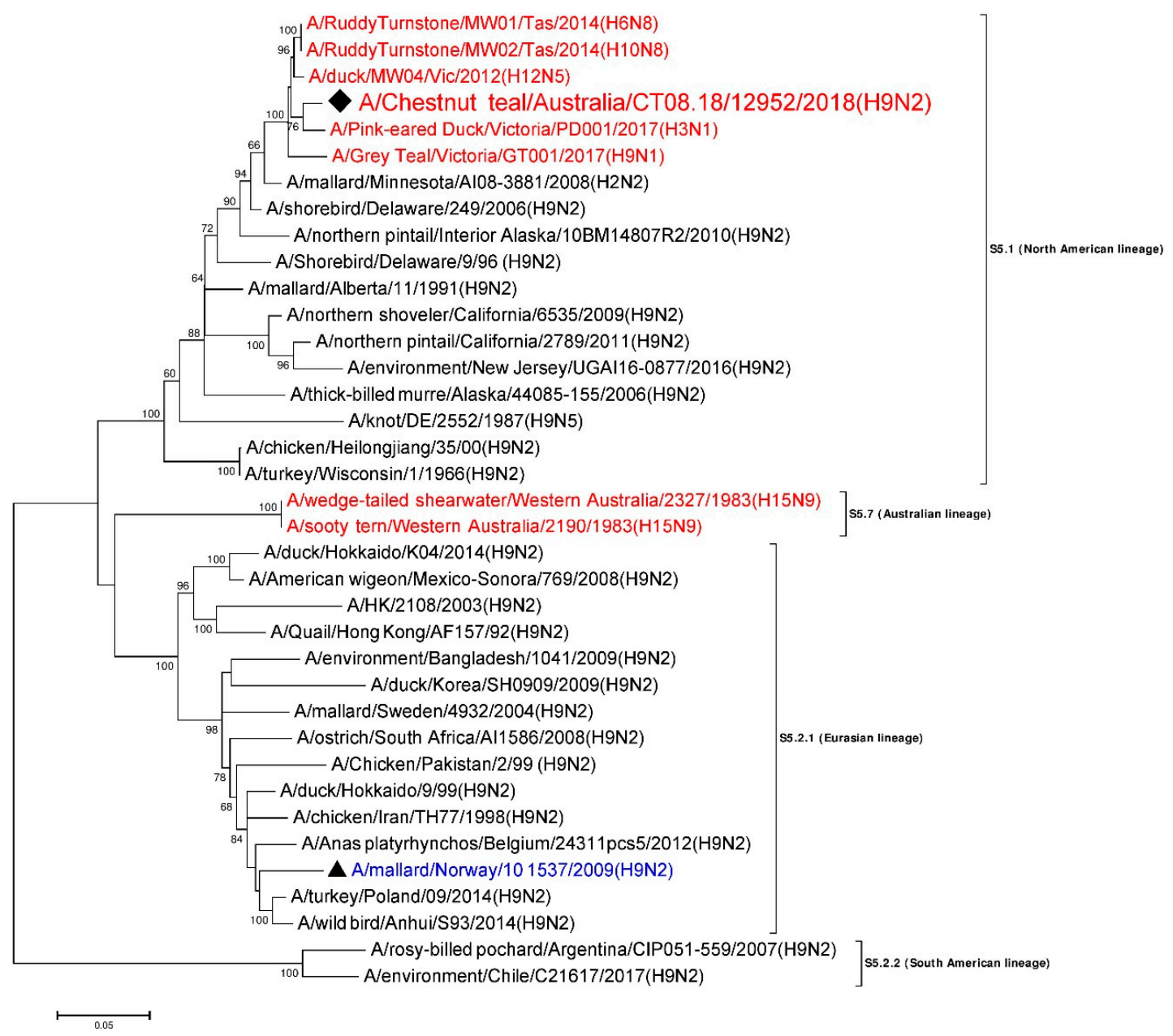

Figure 3. Phylogenetic analysis of the NP gene segment. The nucleotide sequences were aligned and analyzed using the maximum likelihood method in MEGA 7.0 [40], using the General Time Reversible $(\mathrm{GTR}+\mathrm{G}+\mathrm{I})$ [48] model with a bootstrapping of 1000 replicates. The analysis involved 37 sequences of the NP gene segment of influenza virus, including A/Chestnut teal/Australia/CT08.18/12952/2018 (H9N2) from a resident Chestnut teal. The numbers at the nodes represent bootstrap values. Branch lengths are scaled according to the number of nucleotide substitutions per site, and only bootstrap values at or above $60 \%$ are shown. The sequence from the current study has been labelled with a black rhombus $(\downarrow)$, while a representative reference sequence from Eurasian lineage has been labelled with a black triangle ( $\mathbf{\Lambda})$, with text in blue color, and is shown on all figures for easy reference. Red colored sequences are from AIVs detected in Australia. 
3.8. Phylogenetic Analysis and Molecular Characterization of the Remaining 5 Internal Gene Segments of the H9N2 AIV

Phylogenetic analyses of the three internal gene segments (PB2, M1-M2 and NEP-NS1) were conducted using the obtained full gene segment sequences with a coverage depth ranging from 2 to 4634 at a mapping quality threshold of 80 and above from the individual sample 12952. For the gene segments PB1 and PA, where we did not obtain full length sequences, concatenated sequences of 1381nt and 1130nt long, respectively, were assembled from the pooled sample CT08.18 for the phylogenetic analysis. Comparison of the partial sequences of the PB1 and PA gene segments obtained from the individual sample 12952 without PCR amplification to the PB1 and PA from CT08.18, found that they were $100 \%$ identical where they overlapped. Phylogenetic analysis indicated that all 5 gene segments fell within the Eurasian lineage with the PB2 gene segment in sub-lineage S1.2.4, PB1 in S2.2.1, PA in S3.2.7, M1-M2 in S7.2.5 and NEP-NS1 in the S8.2.1 sub-lineages [34,44,47] (Tables 1 and 2 and Supplementary Figures S1-S5).

\subsection{Attempt at Virus Isolation}

The attempt for AIV isolation on the two influenza PCR positive individual samples, in embryonated chicken eggs at the Australian Animal Health Laboratory (AAHL), Geelong, gave a negative result.

\section{Discussion}

In this study, we detected and sequenced an aquatic bird Eurasian sub-lineage h9.3.3-n2.1.6 H9N2 LPAI virus from faecal samples collected from two resident Chestnut teals in southeastern Australia. Based on the sequences generated, we concluded that A/Chestnut teal/Australia/CT08.18/12952/2018 (H9N2) was an H9N2 AIV, with seven out of eight gene segments of Eurasian lineage and an NP gene segment of North American lineage. This AIV is therefore most likely a reassortant, with gene segments from viruses from two different lineages, indicating the intercontinental exchange of viral RNA. Another study of Australian resident wild birds recently demonstrated a similar type of intercontinental reassortment in $\mathrm{H} 9 \mathrm{~N} 1$ and H3N1 AIV [28]. Based on the overall results from our phylogenetic analyses, it appears that the six gene segments other than PB1 and NA have been introduced into Australian resident wild birds sometime in the past, while the Eurasian N2 gene segment (Figure 2) and Eurasian PB1 gene segment (Figure S2) occurred more recently, as they have only been detected in recent wild bird AIV surveillance (after 2015). The viruses we found here are therefore the progeny from two or more AIV reassortment events.

The 100\% identity of the partial sequences of PB1 and PA gene segments from individual sample 12952 and pooled sample CT08.18 on the one hand, and the absence of sequences from these segments in individual sample 11352 on the other, support that the obtained partial, concatenated sequences of PB1 and PA from CT08.18 likely came from bird 12952 only.

The negative results of the virus isolation in embryonated chicken eggs from individual samples may have been because of poor sample quality, as the individual samples had been stored for a year when virus isolation was attempted; alternatively, it may be due to a low level of infectious viral particles present in the samples [49]. A low level of AIV in these samples is consistent with the low number of reads obtained even after virus enrichment (only $0.0004 \%$ of AIV reads in the pool), and even fewer AIV reads in the individual samples not subjected to specific AIV PCR amplification. As most of the AIV reads in the individual samples came from the non-ultracentrifuged samples, we speculate that AIV virions may have deteriorated/dried out during the prolonged storage of the individual faecal samples, and that these reads most likely came from partially protected AIV RNA/nucleoprotein complexes. Nevertheless, it is interesting to note that despite a likely low initial virus content and individual samples having been stored for a year, the protocol, including pre-amplification for full length AIV gene segment sequences using a commercial AIV PCR kit, yielded full-length sequences of six out of eight gene segments. Thus, this method of combined virus enrichment (excluding 
ultracentrifugation), followed by specific AIV amplification and NGS, may be of use for other wild bird AIV investigations, where initial NGS or virus isolation attempts have failed or provided limited sequence information.

Although it is reassuring that the virus detected is a typical aquatic bird Eurasian sub-lineage h9.3.3-n2.1.6 H9N2 LPAI virus [50,51], and not of the sub-lineage h9.4-n2.1.4, that has caused infection in humans [16-18], the presence of a reassortant H9N2 AIV in a resident wild bird, with some segments similar to viruses from countries remote to Australia, clearly indicates that these resident species are not isolated in regards to the global circulation of AIV. Therefore, any AIV lineage, including highly pathogenic viruses, could one day arrive.

\section{Data Availability}

Sequences generated for six full gene segments from the individual 12952 samples have been deposited in NCBI Genbank with accession numbers (MN826600-MN826605). Similarly, accession numbers MN156428-MN156434 represent the two partial, concatenated gene segments from the pooled sample CT08.18. The remaining sequences from the pooled sample CT08.18 have the NCBI Genbank accession number MN156424-MN156427 and MN156435-MN156445. Additional datasets analyzed in the paper can be made available from the authors upon reasonable request.

Supplementary Materials: The following are available online at http://www.mdpi.com/1999-4915/12/1/88/s1. The following are added to this manuscript. Table S1: Partial sequences of the eight gene segments of AIV obtained from A/Chestnut teal/Australia/CT08.18/2018(H9N2). Figure S1: Phylogenetic analysis of the PB2 gene segment. The nucleotide sequences were aligned and analyzed using the maximum likelihood method in MEGA 7.0 [40], using the General Time Reversible (GTR+G+I) [48] model with a bootstrapping of 1000 replicates The analysis involved 37 sequences of the PB2 gene segment of influenza virus, including A/Chestnut teal/Australia/CT08.18/12952/2018 (H9N2) from a resident Chestnut teal. The numbers at the nodes represent bootstrap values, and only bootstrap values at or above $60 \%$ are shown. Branch lengths are scaled according to the number of nucleotide substitutions per site. The sequence from the current study has been labelled with a black rhombus $(\downarrow)$, while a representative reference sequence from Eurasian lineage has been labelled with a black triangle $(\mathbf{\Lambda})$, with text in blue color, and is shown on all figures for easy reference. Red colored sequences are from AIVs detected in Australia. Figure S2: Phylogenetic analysis of the partial PB1 gene segment. The $1381 \mathrm{nt}$ long, partial concatenated sequences were aligned and analyzed using the maximum likelihood method in MEGA 7.0 [40], using the Tamura-Nei (TN93+G) [45] model with a bootstrapping of 1000 replicates. The analysis involved 45 sequences of the PB1 gene segment of influenza virus, including A/Chestnut teal/Australia/CT08.18/2018 (H9N2) from resident Chestnut teals. The numbers at the nodes represent bootstrap values, and only bootstrap values at or above $60 \%$ are shown. Branch lengths are scaled according to the number of nucleotide substitutions per site. The sequence from the current study has been labelled with a black rhombus $(\downarrow)$, while a representative reference sequence from Eurasian lineage has been labelled with a black triangle ( $\mathbf{\Lambda})$, with text in blue color, and is shown on all figures for easy reference. Red colored sequences are from AIVs detected in Australia. Figure S3: Phylogenetic analysis of the partial PA gene segment. The $1130 \mathrm{nt}$ long, partial concatenated sequences were aligned and analyzed using the maximum likelihood method in MEGA 7.0 [40] using the General Time Reversible $(G T R+G)[48]$ model with a bootstrapping of 1000 replicates. The analysis involved 37 sequences of the PA gene segment of influenza virus including A/Chestnut teal/Australia/CT08.18/2018 (H9N2) from resident Chestnut teals. The numbers at the nodes represent bootstrap values, and only bootstrap values at or above $60 \%$ are shown. Branch lengths are scaled according to the number of nucleotide substitutions per site. The sequence from the current study has been labelled with a black rhombus $(\downarrow)$, while a representative reference sequence from Eurasian lineage has been labelled with a black triangle ( $\mathbf{\Lambda})$, with text in blue color, and is shown on all figures for easy reference. Red colored sequences are from AIVs detected in Australia. Figure S4: Phylogenetic analysis of the M1-M2 gene segment. The nucleotide sequences were aligned and analyzed using the maximum likelihood method in MEGA 7.0 [40], using the Kimura 2 parameter $(\mathrm{K} 2+\mathrm{G}+\mathrm{I})$ [52] model, with a bootstrapping of 1000 replicates. The analysis involved 47 sequences of the M1-M2 gene segment of influenza virus, including A/Chestnut teal/Australia/CT08.18/12952/2018 (H9N2) from a resident Chestnut teal. The numbers at the nodes represent bootstrap values, and only bootstrap values at or above $60 \%$ are shown. Branch lengths are scaled according to the number of nucleotide substitutions per site. The sequence from the current study has been labelled with a black rhombus $(\downarrow)$, while a representative reference sequence from Eurasian lineage has been labelled with a black triangle $(\boldsymbol{\Lambda})$, with text in blue color, and is shown on all figures for easy reference. Red colored sequences are from AIVs detected in Australia. Figure S5: Phylogenetic analysis of the NEP-NS1 gene segment. The nucleotide sequences were aligned and analyzed using the maximum likelihood method in MEGA 7.0 [40] using the General Time Reversible (GTR+G) [48] model, with a bootstrapping of 1000 replicates. The analysis involved 42 sequences of the NEP-NS1 gene segment of influenza virus, including A/Chestnut teal/Australia/CT08.18/12952/2018 (H9N2) from a resident Chestnut teal. The numbers at the nodes represent bootstrap values, and only bootstrap values at or above $60 \%$ are shown. Branch lengths are scaled according to the number of nucleotide substitutions per site. 
The sequence from the current study has been labelled with a black rhombus $(\bullet)$, while a representative reference sequence from Eurasian lineage has been labelled with a black triangle ( $\mathbf{\Lambda})$, with text in blue color, and is shown on all figures for easy reference. Red colored sequences are from AIVs detected in Australia.

Author Contributions: S.A. initiated the study and coordinated all work carried out at GCEID. M.K. collected the samples. T.R.B. and J.V. performed the laboratory work. T.R.B., A.C., and S.A. carried out the NGS data analysis. T.R.B. drafted the initial manuscript, and later versions were based on input and suggestions from all. All authors contributed to the final submitted version. All authors have read and agreed to the published version of the manuscript.

Funding: This research was funded by the Australian Government Research Training Program Scholarship through Deakin University; NHMRC Equipment Grant GNT9000413 through Deakin University; and funding provided by Deakin University, Barwon Health, and CSIRO to Soren Alexandersen, with BirdLife Australia funding to Jessy Vibin.

Acknowledgments: We want to acknowledge Trent Leen for providing access to his property for bird-catching, and other members of Geelong Field and Game for providing assistance with catching and sampling of Chestnut teals. Similarly, we want to acknowledge AAHL, Geelong, Australia, for attempting virus isolation from the individual faecal samples. We also want to acknowledge the WHO Collaborating Centre for Reference and Research on Influenza, Melbourne, Australia, for independently doing matrix gene PCR on individual fecal samples. Finally, we acknowledge Jason Hodge, laboratory manager of the GCEID laboratory.

Conflicts of Interest: The authors declare no conflict of interest. The funders had no role in the design of the study; in the collection, analyses, or interpretation of data; in the writing of the manuscript, or in the decision to publish the results.

\section{References}

1. Tong, S.; Zhu, X.; Li, Y.; Shi, M.; Zhang, J.; Bourgeois, M.; Yang, H.; Chen, X.; Recuenco, S.; Gomez, J. New world bats harbor diverse influenza a viruses. PLoS Pathog. 2013, 9, e1003657. [CrossRef] [PubMed]

2. Bouvier, N.M.; Palese, P. The biology of influenza viruses. Vaccine 2008, 26, D49-D53. [CrossRef] [PubMed]

3. Nelson, M.I.; Holmes, E.C. The evolution of epidemic influenza. Nat. Rev. Genet. 2007, 8, 196. [CrossRef] [PubMed]

4. Turner, A. The role of wild aquatic birds in the epidemiology of avian influenza in Australia. Aust. Vet. J. 2004, 82, 713. [CrossRef] [PubMed]

5. Horimoto, T.; Rivera, E.; Pearson, J.; Senne, D.; Krauss, S.; Kawaoka, Y.; Webster, R.G. Origin and molecular changes associated with emergence of a highly pathogenic h5n2 influenza virus in mexico. Virology 1995, 213, 223-230. [CrossRef]

6. Garcia, M.; Crawford, J.M.; Latimer, J.W.; RiveraCruz, E.; Perdue, M.L. Heterogeneity in the haemagglutinin gene and emergence of the highly pathogenic phenotype among recent $\mathrm{h} 5 \mathrm{n} 2$ avian influenza viruses from mexico. J. Gen. Virol. 1996, 77, 1493-1504. [CrossRef]

7. Spackman, E.; Senne, D.A.; Davison, S.; Suarez, D.L. Sequence analysis of recent h7 avian influenza viruses associated with three different outbreaks in commercial poultry in the united states. J. Virol. 2003, 77, 13399-13402. [CrossRef]

8. Banks, J.; Speidel, E.S.; Moore, E.; Plowright, L.; Piccirillo, A.; Capua, I.; Cordioli, P.; Fioretti, A.; Alexander, D.J. Changes in the haemagglutinin and the neuraminidase genes prior to the emergence of highly pathogenic h7n1 avian influenza viruses in italy. Arch. Virol. 2001, 146, 963-973. [CrossRef]

9. Monne, I.; Fusaro, A.; Nelson, M.I.; Bonfanti, L.; Mulatti, P.; Hughes, J.; Murcia, P.R.; Schivo, A.; Valastro, V.; Moreno, A.; et al. Emergence of a highly pathogenic avian influenza virus from a low-pathogenic progenitor. J. Virol. 2014, 88, 4375-4388. [CrossRef]

10. Dietze, K.; Graaf, A.; Homeier-Bachmann, T.; Grund, C.; Forth, L.; Pohlmann, A.; Jeske, C.; Wintermann, M.; Beer, M.; Conraths, F.J. From low to high pathogenicity-Characterization of h7n7 avian influenza viruses in two epidemiologically linked outbreaks. Transbound. Emerg. Dis. 2018, 65, 1576-1587. [CrossRef]

11. Luczo, J.M.; Tachedjian, M.; Harper, J.A.; Payne, J.S.; Butler, J.M.; Sapats, S.I.; Lowther, S.L.; Michalski, W.P.; Stambas, J.; Bingham, J. Evolution of high pathogenicity of h5 avian influenza virus: Haemagglutinin cleavage site selection of reverse-genetics mutants during passage in chickens. Sci. Rep. 2018, 8, 11518. [CrossRef]

12. Alexander, D.J. An overview of the epidemiology of avian influenza. Vaccine 2007, 25, 5637-5644. [CrossRef] [PubMed] 
13. James, J.; Sealy, J.E.; Iqbal, M. A global perspective on h9n2 avian influenza virus. Viruses 2019, 11, 620.

14. Guo, Y.; Krauss, S.; Senne, D.; Mo, I.; Lo, K.; Xiong, X.; Norwood, M.; Shortridge, K.; Webster, R.; Guan, Y. Characterization of the pathogenicity of members of the newly established $\mathrm{h} 9 \mathrm{n} 2$ influenza virus lineages in asia. Virology 2000, 267, 279-288. [CrossRef] [PubMed]

15. Li, S.; Li, X.; Zhong, S.; Sun, H.; Pan, J.; Chen, S.; Peng, D.; Liu, X. Genome sequencing and phylogenetic analysis of avian influenza viruses subtype h9n2. Bing Du Xue Bao Chin. J. Virol. 2012, 28, 7-14.

16. Lin, Y.; Shaw, M.; Gregory, V.; Cameron, K.; Lim, W.; Klimov, A.; Subbarao, K.; Guan, Y.; Krauss, S.; Shortridge, K. Avian-to-human transmission of h9n2 subtype influenza a viruses: Relationship between h9n2 and h5n1 human isolates. Proc. Natl. Acad. Sci. USA 2000, 97, 9654-9658. [CrossRef]

17. Butt, K.; Smith, G.J.; Chen, H.; Zhang, L.; Leung, Y.C.; Xu, K.; Lim, W.; Webster, R.G.; Yuen, K.; Peiris, J.M. Human infection with an avian h9n2 influenza a virus in hong kong in 2003. J. Clin. Microbiol. 2005, 43, 5760-5767. [CrossRef]

18. Potdar, V.; Hinge, D.; Satav, A.; Simões, E.F.; Yadav, P.D.; Chadha, M.S. Laboratory-confirmed avian influenza a (H9N2) virus infection, India, 2019. Emerging Infect. Dis. 2019, 25, 2328. [CrossRef]

19. Gu, M.; Chen, H.; Li, Q.; Huang, J.; Zhao, M.; Gu, X.; Jiang, K.; Wang, X.; Peng, D.; Liu, X. Enzootic genotype $\mathrm{s}$ of h9n2 avian influenza viruses donates internal genes to emerging zoonotic influenza viruses in China. Vet. Microbiol. 2014, 174, 309-315. [CrossRef]

20. Zhao, G.; Gu, X.; Lu, X.; Pan, J.; Duan, Z.; Zhao, K.; Gu, M.; Liu, Q.; He, L.; Chen, J. Novel reassortant highly pathogenic h5n2 avian influenza viruses in poultry in china. PLoS ONE 2012, 7, e46183. [CrossRef]

21. Wu, A.; Su, C.; Wang, D.; Peng, Y.; Liu, M.; Hua, S.; Li, T.; Gao, G.F.; Tang, H.; Chen, J. Sequential reassortments underlie diverse influenza h7n9 genotypes in china. Cell Host Microbe 2013, 14, 446-452. [CrossRef] [PubMed]

22. Sediri, H.; Thiele, S.; Schwalm, F.; Gabriel, G.; Klenk, H.-D. Pb2 subunit of avian influenza virus subtype h9n2: A pandemic risk factor. J. Gen. Virol. 2016, 97, 39-48. [CrossRef] [PubMed]

23. Yuan, R.; Liang, L.; Wu, J.; Kang, Y.; Song, Y.; Zou, L.; Zhang, X.; Ni, H.; Ke, C. Human infection with an avian influenza a/h9n2 virus in guangdong in 2016. J. Infect. 2017, 74, 422-425. [CrossRef] [PubMed]

24. Shanmuganatham, K.K.; Jones, J.C.; Marathe, B.M.; Feeroz, M.M.; Jones-Engel, L.; Walker, D.; Turner, J.; Rabiul Alam, S.; Kamrul Hasan, M.; Akhtar, S. The replication of bangladeshi h9n2 avian influenza viruses carrying genes from h7n3 in mammals. Emerg. Microbes Infect. 2016, 5, 1-12. [CrossRef]

25. Tracey, J.P.; Woods, R.; Roshier, D.; West, P.; Saunders, G.R. The role of wild birds in the transmission of avian influenza for australia: An ecological perspective. Emu 2004, 104, 109-124. [CrossRef]

26. Yoon, S.-W.; Webby, R.J.; Webster, R.G. Evolution and ecology of influenza a viruses. In Influenza Pathogenesis and Control-Volume I; Springer: Berlin/Heidelberg, Germany, 2014; pp. 359-375.

27. Wille, M.; Eden, J.S.; Shi, M.; Klaassen, M.; Hurt, A.C.; Holmes, E.C. Virus-virus interactions and host ecology are associated with rna virome structure in wild birds. Mol. Ecol. 2018, 27, 5263-5278. [CrossRef]

28. Wille, M.; Shi, M.; Klaassen, M.; Hurt, A.C.; Holmes, E.C. Virome heterogeneity and connectivity in waterfowl and shorebird communities. ISME J. 2019, 13, 2603-2616. [CrossRef]

29. Wille, M.; Lisovski, S.; Risely, A.; Ferenczi, M.; Roshier, D.; Wong, F.Y.; Breed, A.C.; Klaassen, M.; Hurt, A.C. Serologic evidence of exposure to highly pathogenic avian influenza h5 viruses in migratory shorebirds, australia. Emerg. Infect. Dis. 2019, 25, 1903. [CrossRef]

30. Norman, F.; Brown, R. Aspects of the distribution and abundance of chestnut teal in south-eastern australia. Emu-Austral Ornithol. 1988, 88, 70-80. [CrossRef]

31. Hansen, B.D.; Menkhorst, P.; Moloney, P.; Loyn, R.H. Long-term declines in multiple waterbird species in a tidal embayment, south-east a ustralia. Austral. Ecol. 2015, 40, 515-527. [CrossRef]

32. Beaumont, L.J.; McALLAN, I.A.; Hughes, L. A matter of timing: Changes in the first date of arrival and last date of departure of australian migratory birds. Glob. Chang. Biol. 2006, 12, 1339-1354. [CrossRef]

33. Liu, S.; Ji, K.; Chen, J.; Tai, D.; Jiang, W.; Hou, G.; Chen, J.; Li, J.; Huang, B. Panorama phylogenetic diversity and distribution of type a influenza virus. PLOS ONE 2009, 4, e5022. [CrossRef] [PubMed]

34. Chen, J.M.; Sun, Y.X.; Chen, J.W.; Liu, S.; Yu, J.M.; Shen, C.J.; Sun, X.D.; Peng, D. Panorama phylogenetic diversity and distribution of type a influenza viruses based on their six internal gene sequences. Virol. J. 2009, 6, 137. [CrossRef] [PubMed]

35. Hansbro, P.M.; Warner, S.; Tracey, J.P.; Arzey, K.E.; Selleck, P.; O’Riley, K.; Beckett, E.L.; Bunn, C.; Kirkland, P.D.; Vijaykrishna, D.; et al. Surveillance and analysis of avian influenza viruses, australia. Emerg Infect Dis. 2010, 16, 1896-1904. [CrossRef] [PubMed] 
36. Bhatta, T.R.; Chamings, A.; Vibin, J.; Alexandersen, S. Detection and characterisation of canine astrovirus, canine parvovirus and canine papillomavirus in puppies using next generation sequencing. Sci. Rep. 2019, 9, 4602. [CrossRef] [PubMed]

37. Vibin, J.; Chamings, A.; Collier, F.; Klaassen, M.; Nelson, T.M.; Alexandersen, S. Metagenomics detection and characterisation of viruses in faecal samples from australian wild birds. Sci. Rep. 2018, 8, 8686. [CrossRef] [PubMed]

38. Altschul, S.F.; Gish, W.; Miller, W.; Myers, E.W.; Lipman, D.J. Basic local alignment search tool. J. Mol. Biol. 1990, 215, 403-410. [CrossRef]

39. Mount, D.W. Using the basic local alignment search tool (blast). Cold Spring Harb. Protoc. 2007, 2007. [CrossRef]

40. Kumar, S.; Stecher, G.; Tamura, K. Mega7: Molecular evolutionary genetics analysis version 7.0 for bigger datasets. Mol. Biol. Evol. 2016, 33, 1870-1874. [CrossRef]

41. Caboche, S.; Audebert, C.; Lemoine, Y.; Hot, D. Comparison of mapping algorithms used in high-throughput sequencing: Application to ion torrent data. BMC Genom. 2014, 15, 264. [CrossRef]

42. Thorvaldsdóttir, H.; Robinson, J.T.; Mesirov, J.P. Integrative genomics viewer (igv): High-performance genomics data visualization and exploration. Brief. Bioinform. 2013, 14, 178-192. [CrossRef] [PubMed]

43. Larkin, M.A.; Blackshields, G.; Brown, N.; Chenna, R.; McGettigan, P.A.; McWilliam, H.; Valentin, F.; Wallace, I.M.; Wilm, A.; Lopez, R. Clustal w and clustal x version 2.0. Bioinformatics 2007, 23, 2947-2948. [CrossRef] [PubMed]

44. Xu, K.M.; Ferreri, L.; Rimondi, A.; Olivera, V.; Romano, M.; Ferreyra, H.; Rago, V.; Uhart, M.; Chen, H.J.; Sutton, T.; et al. Isolation and characterization of an h9n2 influenza virus isolated in argentina. Virus Res. 2012, 168, 41-47. [CrossRef]

45. Tamura, K.; Nei, M. Estimation of the number of nucleotide substitutions in the control region of mitochondrial DNA in humans and chimpanzees. Mol. Biol. Evol. 1993, 10, 512-526. [PubMed]

46. Tamura, K. Estimation of the number of nucleotide substitutions when there are strong transition-transversion and g+c-content biases. Mol. Biol. Evol. 1992, 9, 678-687. [PubMed]

47. Gonzalez-Reiche, A.S.; Perez, D.R. Where do avian influenza viruses meet in the americas? Avian Dis. 2012, 56, 1025-1033. [CrossRef]

48. Nei, M.; Kumar, S. Molecular Evolution and Phylogenetics; Oxford University Press: Oxford, UK, 2000.

49. Eisfeld, A.J.; Neumann, G.; Kawaoka, Y. Influenza a virus isolation, culture and identification. Nat. Protoc. 2014, 9, 2663. [CrossRef]

50. Lindh, E.; Ek-Kommonen, C.; Vaananen, V.M.; Vaheri, A.; Vapalahti, O.; Huovilainen, A. Molecular epidemiology of h9n2 influenza viruses in northern europe. Vet. Microbiol. 2014, 172, 548-554. [CrossRef]

51. Van Borm, S.; Rosseel, T.; Marché, S.; Steensels, M.; Vangeluwe, D.; Linden, A.; van den Berg, T.; Lambrecht, B. Complete coding sequences of one $\mathrm{h} 9$ and three $\mathrm{h} 7 \mathrm{low}$-pathogenic influenza viruses circulating in wild birds in belgium, 2009 to 2012. Genome Announc. 2016, 4, e00540-16. [CrossRef]

52. Kimura, M. A simple method for estimating evolutionary rates of base substitutions through comparative studies of nucleotide-sequences. J. Mol. Evol. 1980, 16, 111-120. [CrossRef]

(C) 2020 by the authors. Licensee MDPI, Basel, Switzerland. This article is an open access article distributed under the terms and conditions of the Creative Commons Attribution (CC BY) license (http://creativecommons.org/licenses/by/4.0/). 